\section{Reducción de volumen pulmonar endoscópica en enfisema pulmonar avanzado. Casos clínicos}

\author{
SEBASTIÁN FERNÁNDEZ-BUSSY ${ }^{1}$, GONZALO LABARCA ${ }^{2,3}$, \\ IVÁN CAVIEDES ${ }^{1}$, HIREN J. MEHTA ${ }^{4}$, MICHAEL JANTZ ${ }^{4}$, ADNAN MAJID ${ }^{5}$
}

\section{Endoscopic lung volume reduction in advanced pulmonary emphysema: initial experience in Chile}

Chronic obstructive pulmonary disease (COPD) has no curative treatment, and in moderate to advanced stages, functional parameters and quality of life are affected. Lung volume reduction improves respiratory parameters and quality of life of these patients. Endoscopic lung volume reduction is a minimally invasive procedure that uses endobronchial valves or coils. Valves are unidirectional, blocking the air from entering the target lobe during inspiration, allowing the exit of air and secretions during expiration. Complete fissure and absence of collateral ventilation are needed for an adequate functioning of endobronchial valves. Endobronchial coils cause mechanical retraction of the lung parenchyma. We report two patients who underwent endoscopic lung volume reduction by endobronchial valves. One patient was on continuous positive pressure non-invasive ventilation due to his severe emphysema.

(Rev Med Chile 2017; 145: 667-672)

Key words: Bronchoscopy; Pulmonary Disease, Chronic Obstructive; Pulmonary Emphysema.

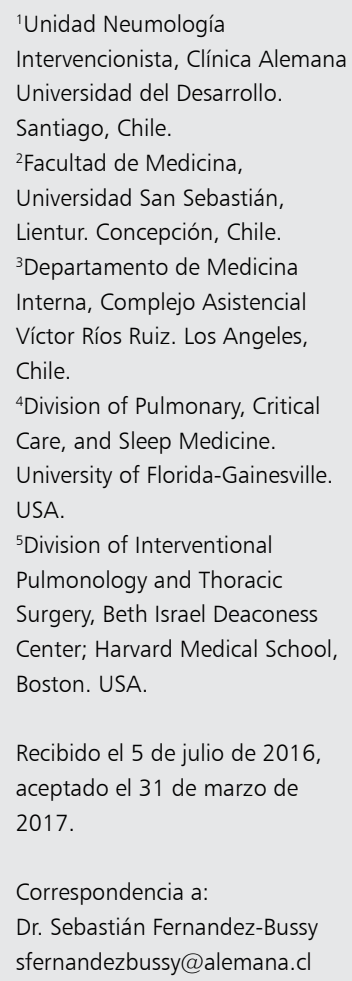

L a enfermedad pulmonar obstructiva crónica (EPOC) es una patología sin tratamiento curativo, que en etapas moderadas a avanzadas afecta tanto los parámetros funcionales como la calidad de vida ${ }^{1}$. En las etapas finales de la enfermedad, y en aquellos enfermos en los cuales el trasplante pulmonar no es planteable (o bien como puente a éste), se han desarrollado nuevas estrategias para mejorar parámetros funcionales y de calidad de vida. Dentro de éstas se encuentra la reducción de volumen pulmonar quirúrgica y la reducción de volumen pulmonar endoscópica (RVPE), siendo esta última técnica un procedimiento mínimamente invasivo ${ }^{2}$. Los candidatos a reducción de volumen quirúrgica deben cumplir similares características funcionales que los candidatos a reducción de volumen endoscópica. Una diferencia a destacar, es que la reducción quirúrgica sólo se efectúa en lóbulos superiores, mientras que la RVPE puede ser en lóbulos superiores e inferiores. La RVPE fue descrita inicialmente el año 2003 y pueden ser agrupadas en técnicas de RVPE de bloqueo como válvulas endobronquiales (VE) o RVPE de no-bloqueo (tales como coils, ablación térmica por vapor, e instilación de polímeros). Por otro lado, existen las que son definitivas o no reversibles (coils y ablación por vapor), y la técnica reversible de $\mathrm{VE}^{2,3}$.

Dentro de los criterios de inclusión de estas técnicas, es relevante hacer mención que sólo son planteables en pacientes mayores de 35 años, sin antecedente de tabaquismo activo, portadores de enfisema pulmonar avanzado asociado a un volumen espiratorio forzado 1 segundo $\left(\mathrm{VEF}_{1}\right)$ 
Tabla 1. Comparación entre válvulas y coils endobronquiales

\begin{tabular}{|c|c|c|}
\hline & Válvulas & Coil \\
\hline Mecanismo de acción & Atelectasia lobar & $\begin{array}{l}\text { Compresión de parénquima mediante reducción } \\
\text { del lóbulo objetivo }\end{array}$ \\
\hline Reversibilidad & Reversible & Parcialmente irreversible \\
\hline Pre requisitos & $\begin{array}{l}V E F_{1} 45-50 \% \\
V R>150 \% \\
\text { Enfisema heterogéneo con cisuras completa }\end{array}$ & $\begin{array}{l}V E F_{1}<45 \% \\
V R>175 \% \\
\text { Enfisema heterogéneo u homogéneo }\end{array}$ \\
\hline Ventilación colateral & Dependiente & Independiente \\
\hline Complicaciones & Neumotórax & $\begin{array}{l}\text { Hemoptisis } \\
\text { Reacción inflamatoria }\end{array}$ \\
\hline
\end{tabular}

bajo $45-50 \%$ del predicho y a un volumen residual (VR) mayor de $150 \%$, junto a una baja capacidad de ejercicio ${ }^{3}$.

Una vez seleccionado el paciente, se debe realilizar estudio con imágenes para evaluar la presencia y características del enfisema, así como la integridad de las cisuras y la presencia o ausencia de ventilación colateral. El TAC de tórax de alta resolución ayuda a definir pacientes candidatos a la intervención y predecir el éxito de la misma². En la Tabla 1 se muestran las principales diferencias entre válvulas y coils.

\section{Reporte de casos}

\section{Caso 1}

Hombre de 69 años, con diagnóstico de EPOC y enfisema severo, $\operatorname{VEF}_{1} 0,58$ Lts $(25,1 \%)$ y VR 5,4 Lts (216\%), con índice paquete/año (IPA) de 45. Fue hospitalizado por cuadro de disnea progresiva, aumento de requerimientos de oxígeno hasta llegar a requerir VMNI de manera continua por insuficiencia respiratoria global. Se solicitó evaluación para RVPE. La radiografía $(\mathrm{Rx})$ y TAC de tórax evidenció enfisema severo heterogéneo ade predominio en el lóbulo inferior derecho (LID) (Figura 1A y 1B) con una cisura mayor aparentemente completa. Se efectuó evaluación de la ventilación colateral endoscópica por sistema CHARTIS $^{\circledR}$, que certificó la ausencia de ventilación colateral. Se instalaron tres VE (Zephyr $\left.{ }^{\circledR}\right)$ en LID (Figura 2A y B). Presentó neumotórax masivo del pulmón derecho $36 \mathrm{~h}$ post procedimiento. Se instaló drenaje pleural 14 French por técnica de
Seldinger percutánea, el que fue retirado a los 3 días. No fue necesario la extracción de ninguna válvula. La radiografía tórax de control al alta (6 días post colocación de válvulas) demostró re expansión pulmonar derecha completa (Figura 1C y 1D). A los 6 meses de seguimiento, presenta $\mathrm{VEF}_{1}$ 0,87 Lts (37,7\%), VR 4,6 Lts (184\%), escala de St. George con cambio de 68 a 31 puntos, caminando $378 \mathrm{mts}$ en el test de caminata en 6 min (TC6M) (previamente no era capaz de realizar la prueba). Clínicamente sólo requiere oxígeno suplementario durante el ejercicio.

\section{Caso 2}

Hombre de 63 años, con antecedentes de enfisema pulmonar severo con historia de tabaquismo suspendido con IPA de 35, en tratamiento con anticolinérgicos y beta agonistas de acción prolongada y rehabilitación pulmonar. Fue referido para evaluación de VE desde otro centro. Rx de tórax mostró hiperinsuflación pulmonar (Figura $3 \mathrm{~A}$ ), lo que fue confirmado con TAC de tórax que evidenció enfisema pulmonar heterogéneo de predominio en ambos lóbulos superiores con cisura mayor izquierda de 90\%. Evaluación por sistema VIDA ${ }^{\circledR}$ sobre integridad de las cisuras, demostró cisura izquierda de 100\% (Figura 4). Esto fue confirmado por ausencia de ventilación colateral con sistema CHARTIS ${ }^{\circledR}$. Parámetros de función pulmonar basales mostraron un $\mathrm{VEF}_{1}$ de 1,13 Lts (48,3\% del predicho), volumen residual de 4,70 Lts (188,1\% del predicho), cuestionario St. George de 65 puntos y TC6M de $232 \mathrm{mts}$. Se instalaron tres VE en lóbulo superior izquierdo, sin complicaciones post procedimiento (Figura 3B). 
CASO CLÍNICO
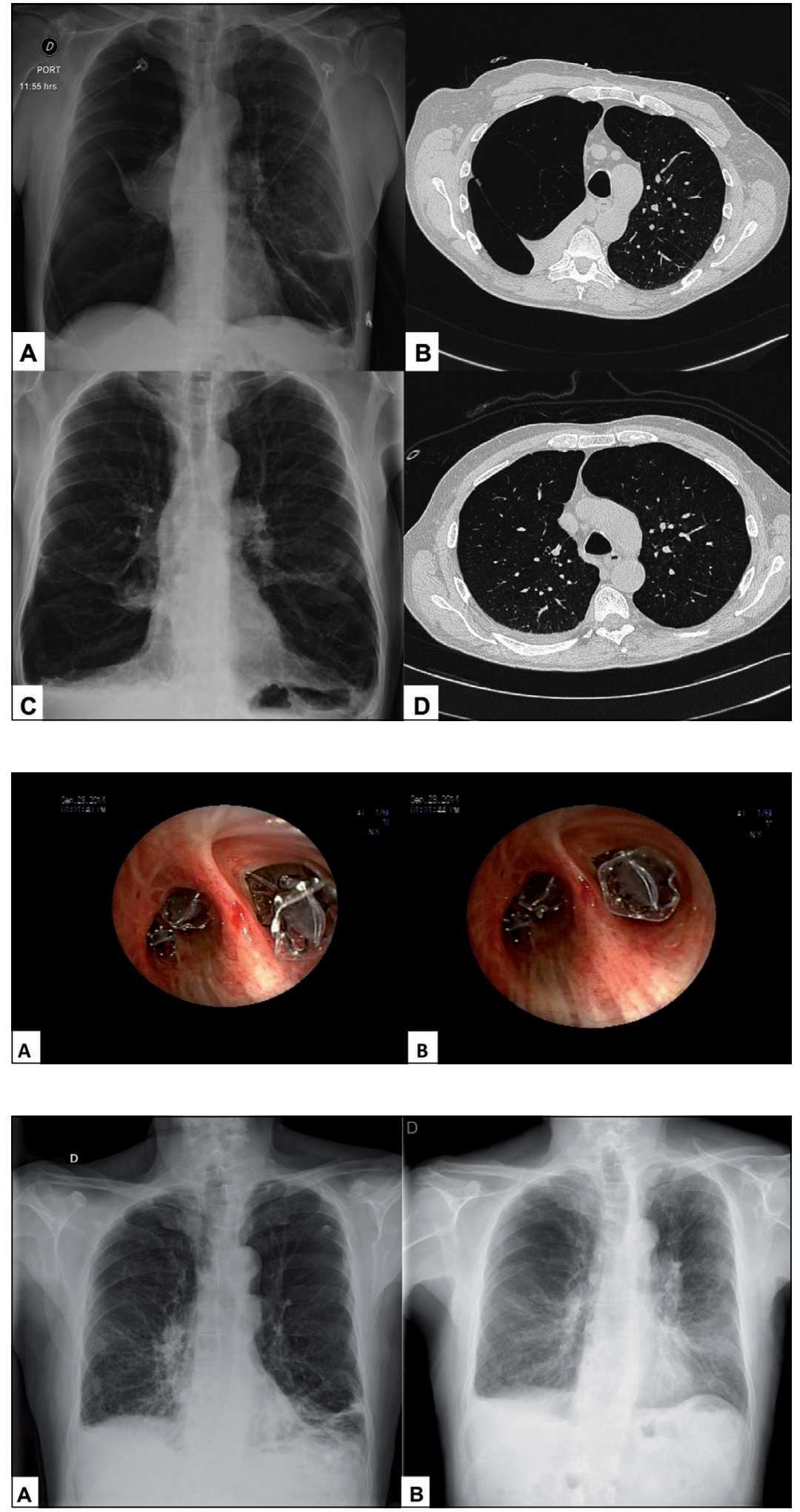

Figura 1. A y B: Enfisema heterogéneo a predomino del lóbulo inferior derecho con cisura completa; C y D: Retracción del lóbulo inferior derecho con expansión del lóbulo medio y superior derecho.

Figura 2. A y B: Válvulas Zephyr en segmento apical, y tronco de segmentos basales del lóbulo inferior derecho.

Figura 3. Radiografía de tórax de paciente con EPOC. A) Hiperinsuflación pulmonar bilateral; B) Posterior a instalación de 3 válvulas endobronquiales en el lóbulo superior izquierdo, se aprecia retracción del mismo, con expansión del lobar inferior izquierdo, aumento de la trama pulmonar y elevación del hemidiafragma. 


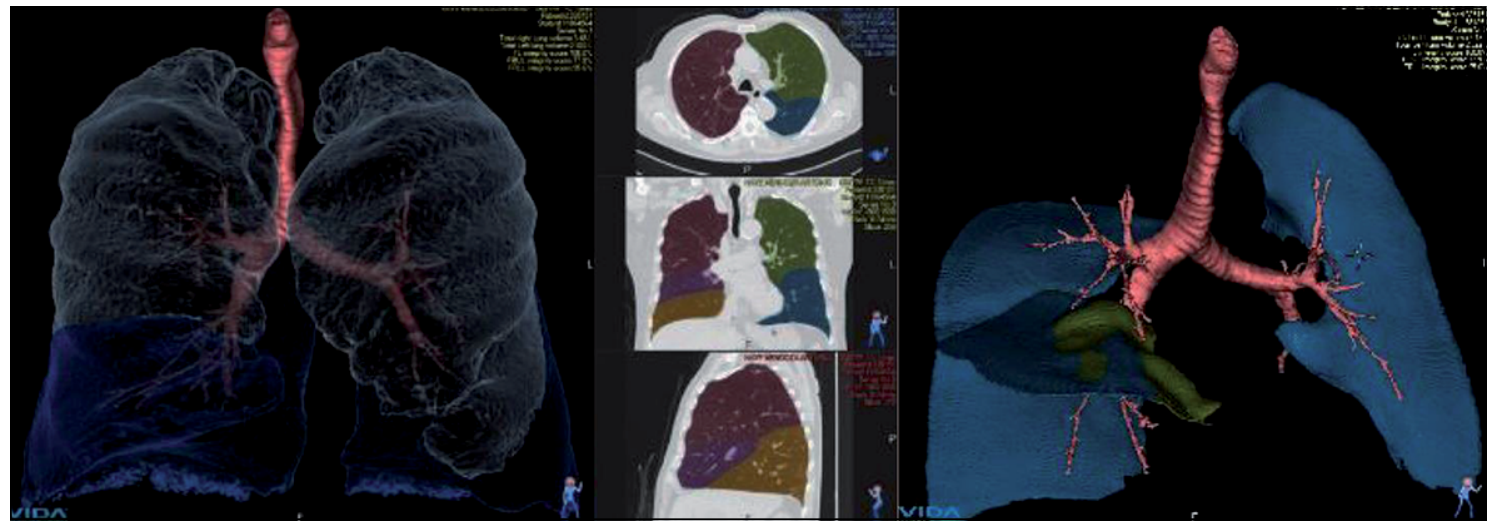

Figura 4. Sistema VIDA en la evaluación de integridad de las cisuras.

A los 6 meses de seguimiento presenta aumento de TC6M en $62 \mathrm{mts}$, aumento del $\mathrm{VEF}_{1}$ a 1,35 Lts $(57,7 \%)$, con reducción del volumen residual a 3,90 Lts $(156 \%)$ y del puntaje de cuestionario St. George a 53 puntos. Presentando clínicamente disnea mínima al ejercicio.

\section{Discusión}

La reducción de volumen pulmonar endoscópica por VE consiste en la instalación de válvulas endobronquiales unidireccionales que bloquean el ingreso de aire hacia el lóbulo objetivo, pero que permiten su salida. Esto permite que dicho lóbulo se vaya desinflando durante espiraciones sucesivas hasta llegar a la atelectasia lobar total. Para ello es necesario realizar una oclusión lobar completa, cubriendo todos los bronquios segmentarios con válvulas, y las cisuras interlobares deben estar completas. La integridad de las cisuras puede ser evaluado con tomografía de alta resolución y diferentes softwares computacionales como el sistema VIDA ${ }^{\circledR 4}$. Por otro lado, el sistema CHARTIS ${ }^{\circledR}$ permite una evaluación endoscópica de la ventilación colateral ${ }^{5}$. Es imprescindible que exista ausencia de ventilación colateral para que el tratamiento con válvulas sea efectivo. Hoy en día existen dos tipos de válvulas comercialmente disponibles: VE compuestas de nitinol y silicona (Zephyr ${ }^{\circledR}$; Pulmonx. Suiza) y válvulas intrabronquiales compuestas por un esqueleto de nitinol en forma de paraguas cubierto de una membrana de poliuretano $\left(\mathrm{IBVs}^{\circledR} \text {; Olympus. Japón }\right)^{3}$.
Existen a la fecha cuatro estudios clínicos aleatorizados que evalúan la eficacia y seguridad de las VE. En el estudio VENT, se aleatorizaron 220 pacientes con enfisema a válvulas versus manejo médico convencional. Tras un plazo de 6 meses se obtuvo un aumento del $\mathrm{VEF}_{1}$ de $6,8 \%$ y una diferencia en el test de caminata de 6 min de 5,8\% del total de metros recorridos, reportando una mejoría en calidad de vida (todo estadísticamente significativos). Se reportó $10,3 \%$ de complicaciones asociadas al procedimiento en el grupo VE y $3,9 \%$ en el grupo manejo convencional (sin significancia estadística). Del total de eventos adversos, la exacerbación de la EPOC fue la más frecuente $9,3 \%$, registrándose hemoptisis leve en $6,1 \%$ y neumotórax en 4,2\% del total de los pacientes ${ }^{6}$. Análisis posteriores demostraron que en aquellos pacientes con oclusión completa del lóbulo afectado y una cisura interlobar mayor de $90 \%$ (cisura entre el lóbulo tratado y los adyacentes en al menos un eje) tienen un mayor beneficio ${ }^{7-9}$. Pacientes con estos criterios presentaron un aumento de $26 \% \pm$ $24 \%$ del $\mathrm{VEF}_{1}$ y un aumento del $22 \% \pm 38 \%$ de TC6M en el seguimiento de la cohorte Euro-VENT a 6 meses 9 .

Una de los principales desafíos es definir aquellos pacientes que van a presentar una cisura interlobar completa, ausencia de ventilación colateral y que se beneficiarían de VE. Existen a la fecha modelos de predicción basados en tomografía axial computada y mediante sistema de medición de cisura con software predictivos (VIDA System ${ }^{\circledR}$, Brasil). En ambos métodos se puede medir el porcentaje de cisura a nivel del lóbulo target. Se 
definen como lóbulos no candidatos a válvulas a aquellos que presentan integridad de cisura menor a $75 \%$, mientras que, ante una integridad entre 75 $90 \%$, se necesitan estudios complementarios para tomar la decisión. Aquellos con cisuras mayores a $90 \%$ son candidatos a VE. En aquellos casos en los que no es posible predecir mediante TAC o ante cisuras entre $75-90 \%$, se recomienda realizar la medición de la ventilación colateral mediante catéter oclusivo durante la broncoscopía (Chartis ${ }^{\circledR}$. Pulmonx, Suiza). Este método permite evaluar la presencia de ventilación colateral mediante la oclusión con balón del lóbulo target ${ }^{5}$. Se han evaluado ambos métodos concluyendo que permiten seleccionar de manera equivalente pacientes candidatos a VE con una sensibilidad de $75 \%{ }^{10}$.

El 2015 se publicaron los resultados del estudio BeLieVer-HIFI trial. En él se aleatorizaron pacientes con cisura interlobar intacta mediante TAC de tórax, 25 de ellos fueron al grupo de válvulas y 25 a procedimiento placebo. A los 3 meses de seguimiento se encontró un aumento de $24 \%$ del $\mathrm{VEF}_{1}$ en el grupo válvulas mientras que el grupo placebo fue de 3,9\%. También se encontró diferencia significativa en la distancia al TC6M. Otros parámetros secundarios como calidad de vida y volumen residual no mostraron diferencia significativa. Se registraron 2 muertes en el estudio ( 1 por neumotórax atribuido a la instalación de la válvula y otro por evolución del EPOC con cor pulmonar) ${ }^{11}$. Sólo 35\% presentó atelectasia completa del lóbulo intervenido, mientras que $35 \%$ no presentó cambios, lo que abre la interrogante sobre un método más certero de selección de pacientes ${ }^{11}$.

Klooster y cols., a finales de 2015 publicaron los resultados de su estudio clínico aleatorizado. En este estudio, 84 pacientes fueron tratados con VE mientras que 84 fueron a terapia médica óptima. El período de seguimiento fue de 6 meses y los cambios reportados como resultados fueron una mejoría de 17,9\% del $\mathrm{VEF}_{1}$ (IC 7,6-28\%; $\mathrm{p}=0,001)$, la capacidad vital funcional mejoró $14,4 \%$ (IC 4,4-23\%; $\mathrm{p}=0,01$ ) y la diferencia en el TC6M fue de $74 \mathrm{mts}$ (IC 47-100 metros) entre los distintos grupos. Otros resultados como el puntaje en el cuestionario de St. George, el clinical COPD questionnarie (CCQ) y volumen residual también fueron significativos a favor del grupo de válvulas. Dentro de los análisis de subgrupos, pacientes con enfisema homogéneo (53\%) y heterogéneo (47\%) presentaron mejoría significativa, datos concordantes con el análisis posterior de VENT trial. Estos resultados aumentarían las indicaciones de válvulas, siendo la circulación colateral el motivo de exclusión, pero no así el tipo de enfisema9 ${ }^{9}$. Como complicaciones, se reportaron 23 eventos en el grupo de válvulas y 5 en el grupo control $(\mathrm{p}=0,001)$; siendo la presencia de neumotórax la más frecuente ( 6 en grupo válvulas contra 0 en manejo médico $)^{12}$. Recientemente se publicaron los resultados del estudio IMPACT, en el que se aplicaron válvulas en pacientes con enfisema homogéneo y ausencia de ventilación colateral demostrando que el grupo sometido a VE presentó una diferencia del $\mathrm{VEF}_{1}$ de $17 \%$ a los tres meses, así como mejoría en calidad de vida y tolerancia al ejercicio ${ }^{13}$.

Respecto a las complicaciones asociadas a válvulas, a medida que se restringe la inclusión a pacientes sin ventilación colateral, se ha visto un aumento considerable en el número de neumotórax. En estudios posteriores de carácter observacional a largo plazo, se reporta que la incidencia de neumotórax llega hasta $23 \%$, por lo que se recomienda manejar a estos pacientes con monitorización por 48-72 h y utilizar con inhibidores de la tos ${ }^{14-16}$. En un estudio retrospectivo, de 429 pacientes, 25 de ellos (5,9\%) presentó neumotoráx, siendo mayor a 7 días en $68 \%$. Sin embargo, aquellos que presentaron la complicación tuvieron un beneficio en función pulmonar y calidad de vida, por lo que presentar esta complicación no perjudicaría los resultados a largo plazo ${ }^{16}$.

Como conclusión, en estos dos casos y con literatura que lo apoya, la reducción de volumen pulmonar endoscópica mediante VE es una alternativa planteable en pacientes con enfisema pulmonar avanzado, heterogéneo en los que se ha demostrado ausencia de ventilación colateral y volumen residual elevado. Sin embargo, falta más evidencia sobre pacientes con enfisema homogéneo y trabajos de eficacia en sobrevida.

\section{Referencias}

1. Global strategy for the diagnosis mapoC. Global Initiative for Chronic Obstructive Lung Disease (GOLD). 2015 [citado el 2 de junio de 2016].

2. Mulhall P, Criner G. Non-pharmacological treatments for COPD. Respirology 2016. 
3. Gompelmann D, Herth FJ. Novel Technologies in Endoscopic Lung Volume Reduction. Thorac Surg Clin 2016; 26: 177-86.

4. Milanese G, Silva M, Sverzellati N. Lung volume reduction of pulmonary emphysema: the radiologist task. Curr Opin Pulm Med 2016; 22: 179-86.

5. Shah PL, Herth FJ. Dynamic expiratory airway collapse and evaluation of collateral ventilation with Chartis. Thorax 2014; 69: 290-1.

6. Sciurba FC, Ernst A, Herth FJ, Strange C, Criner GJ, Marquette $\mathrm{CH}$, et al, Group VSR. A randomized study of endobronchial valves for advanced emphysema. N Engl J Med 2010; 363: 1233-44.

7. Ninane V, Geltner C, Bezzi M, Foccoli P, Gottlieb J, Welte T, et al. Multicentre European study for the treatment of advanced emphysema with bronchial valves. Eur Respir J 2012; 39: 1319-25.

8. Schuhmann M, Raffy P, Yin Y, Gompelmann D, Oguz I, Eberhardt R, et al. Computed tomography predictors of response to endobronchial valve lung reduction treatment. Comparison with Chartis. Am J Respir Crit Care Med 2015; 191: 767-74.

9. Herth FJ, Noppen M, Valipour A, Leroy S, Vergnon JM, Ficker JH, et al, International VSG. Efficacy predictors of lung volume reduction with Zephyr valves in a European cohort. Eur Respir J 2012; 39: 1334-42.

10. Gompelmann D, Eberhardt R, Slebos DJ, Brown MS, Abtin F, Kim HJ, et al. Diagnostic performance comparison of the Chartis System and high-resolution computerized tomography fissure analysis for planning endoscopic lung volume reduction. Respirology 2014; 19: 524-30.

11. Davey C, Zoumot Z, Jordan S, McNulty WH, Carr DH, Hind MD, et al. Bronchoscopic lung volume reduction with endobronchial valves for patients with heterogeneous emphysema and intact interlobar fissures (the BeLieVeR-HIFi study): a randomised controlled trial. Lancet 2015; 386: 1066-73.

12. Klooster K, ten Hacken NH, Hartman JE, Kerstjens HA, van Rikxoort EM, Slebos DJ. Endobronchial Valves for Emphysema without Interlobar Collateral Ventilation. N Engl J Med 2015; 373: 2325-35.

13. Valipour A, Slebos DJ, Herth F, Darwiche K, Wagner $\mathrm{M}$, Ficker JH, et al. Endobronchial Valve Therapy in Patients with Homogeneous Emphysema: Results from the IMPACT Study. Am J Respir Crit Care Med 2016.

14. Herzog D, Poellinger A, Doellinger F, Schuermann D, Temmesfeld-Wollbrueck B, Froeling V, et al. Modifying Post-Operative Medical Care after EBV Implant May Reduce Pneumothorax Incidence. PLoS One 2015; 10: e0128097.

15. Choi M, Lee WS, Lee M, Jeon K, Sheen S, Jheon S, et al. Effectiveness of bronchoscopic lung volume reduction using unilateral endobronchial valve: a systematic review and meta-analysis. Int J Chron Obstruct Pulmon Dis 2015; 10: 703-10.

16. Gompelmann D, Herth FJ, Slebos DJ, Valipour A, Ernst A, Criner GJ, et al. Pneumothorax following endobronchial valve therapy and its impact on clinical outcomes in severe emphysema. Respiration 2014; 87: 485-91. 